\title{
El reflejo de una tradición: feminización de la vida familiar
}

Ma Ángeles Hernández-Prados - Universidad de Murcia Mㄴa Luisa Belmonte - Universidad de Murcia

Begoña Lara Guillen - Universidad de Murcia

Recepción: 25.05.2020 | Aceptado: 30.11.2020

Correspondencia a través de ORCID: Maㅡ Luisa Belmonte

Citar: Hernández-Prados, MA., Belmonte, ML. y Lara Guillen, B. (2020). El reflejo de una tradición: feminización de la vida familiar. REIDOCREA, 9, 232-243.
$0000-0002-3617-215 X$

0000-0002-1475-3690

0000-0002-3617-215X

Resumen: La participación equilibrada entre mujeres y hombres en la vida familiar, a fin de lograr una igualdad de oportunidades laborales y romper con los estereotipos tradicionales, aún continúa siendo una utopía en muchos hogares. La presente investigación cuantitativo-evaluativa no experimental exploratoria muestra, a través de un cuestionario para recoger la percepción del alumnado acerca de las tareas del hogar en el núcleo doméstico, los roles asumidos por los miembros de la familia, valorando la implicación e interés de los padres por la educación, diferenciando entre la opinión según el género del alumno, junto con edad y nivel de estudios de los padres. Los resultados muestran que, aunque a juicio del estudiante los progenitores se interesan bastante por la educación, son las madres quienes lo hacen en mayor medida. Esta figura materna se erige como centro de planificación de tareas, cuidado de pequeños, mayores o dependientes, mantenimiento, organización y provisión de recursos en el hogar, junto con limpieza y asignación de tareas domésticas. Pese a ello, el liderazgo y la toma de decisiones son atribuidas principalmente al padre, revelando una perspectiva de pensamiento clásico de las generaciones jóvenes, a favor de posiciones en desequilibrio con respecto a las madres, concediendo la autoridad al rol paterno.

Palabras clave: Corresponsabilidad

\section{The reflection of a tradition: feminization of family life}

Abstract: The balanced participation of women and men in family life, in order to achieve equal employment opportunities and break with traditional stereotypes, still continues to be a utopia in many households. The present exploratory non-experimental quantitative-evaluative research shows, through a questionnaire to collect the perception of the students about the tasks of the home in the domestic nucleus, the roles assumed by the members of the family, assessing the involvement and interest of the parents by education, differentiating between the opinion according to the gender of the student, along with age and educational level of the parents. The results show that, although in the student's opinion the parents are very interested in education, it is the mothers who do it to a greater extent. This maternal figure stands as a center for homework planning, caring for children, the elderly or dependents, maintenance, organization and provision of resources at home, along with cleaning and assigning household chores. Despite this, leadership and decision-making are mainly attributed to the father, revealing a classical thinking perspective of the young generations, in favor of positions in imbalance with respect to the mothers, granting authority to the paternal role.

Key words: Co-responsibility

\section{Introducción}

Conciliar el tiempo vital es una cuestión de encaje de bolillo, en la que la relación existente entre la familia y la vida económica resulta particularmente significativa. Según Fernández Sangrador (2008), el origen de la economía se sitúa en el hogar, entendido como unidad de producción y centro de vida, dependiendo el dinamismo de la vida económica de las redes de intercambios de bienes y servicios que involucran a las familias, a través de los vastos recursos de solidaridad que posee. Pero lejos de mantener esta relación asociativa, lo que impera es la dicotomía entre economía, vinculada al mercado laboral remunerado, y la familia, asociada al trabajo solidario, en la que desafortunadamente, "el reconocimiento social se centra en el mercado monetario y el trabajo asalariado, mientras que el trabajo doméstico, realizado en su 
mayor parte por mujeres, queda sin reconocimiento social" (Cerri y Alamillo Martínez, 2012, p.4).

Esta diferenciación de lo público-privado, laboral-familiar, ha marcado tendencia en las sociedades actuales, y, por ende, en sus ciudadanos, quienes acaban anteponiendo lo laboral a lo familiar, y postergando cada vez más la llegada de los hijos. Las razones de la creciente reducción del número de hijos y del retraso del momento de tenerlos son principalmente de índole económico, aunque también se debe a valores sociales e ideológicos, pues el nacimiento de un hijo conlleva "un cambio importante en tiempos y responsabilidades; marca un antes y un después en cuanto a la organización cotidiana de los tiempos, en cuanto a las posibilidades de movilidad y en cuanto a la libertad" propia (Alberdi, 2014, p.14). En definitiva, la multifuncionalidad, la sobreaceleración, y los estilos de vida del siglo XXI marcados por el capitalismo, han reducido considerablemente el tiempo en el hogar.

Pese a la actual reducción, asociada a la incorporación tardía de la mujer al ámbito laboral, aún continúa predominando una diferenciación en el tiempo de permanencia y funcionalidad en el hogar según el género, marcada principalmente por la tradición cultural del patriarcado y las oportunidades de acceso al mercado laboral. De modo que, "el número de horas que una mujer destina al trabajo remunerado está condicionado por el tiempo que dedica a la producción de bienes y servicios dentro del hogar y al cuidado de los integrantes de este" (Sánchez Vargas, Herrera Merino y Perrotini Hernández, 2015, p.652). Esta percepción cultural ha sido asumida por el mundo laboral, de modo que el trabajador más productivo es aquel que no tiene responsabilidades familiares y se dedica a tiempo completo (fulltime) a los intereses de la empresa (Royo, 2011). De modo que al ser las mujeres quienes en la mayoría de los casos asumen la responsabilidad del cuidad de menores y familiares, así como el trabajo no remunerado del hogar, se genera el llamado "techo de cristal" que dificulta el ascenso a puestos de responsabilidad y desenvolvimiento adecuado de la mujer en el puesto laboral (Región de Murcia, 2019).

Para favorecer las oportunidades de trabajo de ambos miembros de la pareja, se contempla la delegación de las tareas domésticas en personas externas. En este sentido, las tareas del hogar forman parte de la reproducción social y cuanto menor es la disposición de los miembros familiares a invertir su tiempo a la realización de estas, mayor es la demanda de trabajo del hogar remunerado mediante la contratación de personas externas (Garavito, 2018). Los motivos señalados por Devetter, (2013) para contratación de personal doméstico son principalmente: el principio de especialización fundado sobre la diferenciación de ingresos, la negociación en las parejas en un intento de garantizar la igualdad de género en las unidades familiares y la voluntad de delegar las tareas juzgadas como ingratas. De modo que el "trabajo de las empleadas domésticas es el que delegan las mujeres de clase media y alta, para poder salir a trabajar y lo desarrollarse en su profesión" (De Dios, 2019, p.86).

Ahora más que nunca se hace necesario externalizar la distribución de las tareas domésticas y los mecanismos de socialización de estas hacia las nuevas generaciones, pues mostrar que aún existen implantadas en los hogares prácticas desigualitarias permite no relajar las acciones educativas esenciales para concienciar prevenir y transformar culturalmente esta realidad. El presente trabajo parte de la hipótesis de que la percepción que los diferentes miembros de la unidad familiar de las tareas del hogar son diferentes, condicionando los patrones actitudinales-comportamentales respecto a las mismas. 


\section{Las tareas del hogar. Revisión de las investigaciones previas}

Las tareas del hogar y el cuidado de los hijos es uno de los temas socioeconómicos protagonista de los últimos años, cosechando multitud de estudios que según la revisión teórica realizada por Aspiazu (2014), permiten concluir que la dificultad de conciliación entre trabajo remunerado y no remunerado afecta profundamente a las mujeres, y que los avances normativo-legislativos sobre la igualdad de género no han sido suficientes.

Las tareas domésticas se definen según Rodríguez Menéndez, Peña y Torío (2010) en base a tres categorías fundamentales: las tareas del hogar que hacen referencia principalmente a la limpieza, reparación y mantenimiento de la vivienda, el cuidado de los hijos e hijas y de otras personas dependientes presentes en la unidad familiar, y el trabajo emocional que aporta bienestar de los miembros familiares y mejora del clima convivencial. Los quehaceres del hogar y el cuidado de los niños y niñas son tareas domésticas que recaen todavía bajo la responsabilidad exclusiva de la mujer, a pesar de que trabaje a la par de su compañero varón (De Dios, 2019).

Esta desigualdad de género respecto a las tareas del hogar adquiere diferentes matices o enfoques. En primer lugar, respecto al tiempo invertido en las labores domésticas, los estudios señalan que, en comparación con los hombres, las mujeres españolas duplican, y en ocasiones hasta triplican el tiempo dedicado a las tareas domésticas (Instituto de la Mujer, 2013), lo que puede tener consecuencias negativas en la conciliación del trabajo y la vida familia, y el sentimiento de sobrecarga (Altintas y Sullivan, 2016). Generalmente, existe una feminización del hogar, hasta el punto de que las parejas en las que solo trabaja la mujer son inusuales y representan una asignación más igualitaria de roles en el hogar (García Román, 2020).

El tiempo en el hogar se vive diferente entre hombres y mujeres, tanto a nivel cuantitativo respeto a la cantidad de presencialidad en el mismo, como en la funcionalidad y el tipo de actividad a realizar durante la permanencia en casa. Se considera que los avances científico-sociales han matizado, pero no han acabado con las desigualdades en cuanto a las responsabilidades asociadas al cuidado doméstico y tareas en el hogar, lo que nos lleva a cuestionarnos si la responsabilidad familiar es una cuestión de género, recayendo principalmente en la figura femenina (Hernández Prados y Lara Guillen, 2015). A pesar del volumen de estudios y enfoques teóricos planteados, la diferenciación de género en cuanto a la cantidad de tiempo invertido en las tareas domésticas no ha sido resuelto, cuestionando críticamente aspectos metodológicos y la fuerte influencia de la respuesta en base a la atribución más o menos equitativa que tienen del género, principalmente en los hombres (Flaquer et al., 2019).

En consonancia, también se ha evidenciado, en segundo lugar, la desigualdad de género en cuanto al volumen de tareas que asumen. De modo que las madres y las hijas tienen una carga más pesada, mostrando una división desequilibrada del trabajo doméstico en función del género, tanto en la relación de pareja como en la de hermanos (Malonda, Tur Porcar y Llorca, 2017). Además, el reparto del trabajo doméstico está relacionado significativamente con el bienestar de la pareja, ya que son la principal fuente de conflicto marital (Altintas y Sullivan, 2016). También tiene efectos negativos en las nuevas generaciones, ya que se relaciona con actitudes sexistas hostiles y benevolentes (Malonda, Tur Porcar y Llorca, 2017).

Por otra parte, el reparto de tareas no se asume de forma pasiva o responsable, ni está cerrada permanentemente, sino que es el resultado de los procesos de negociación marital que definen lo posible y deseable para cada uno de los géneros en base a la acumulación de microdecisiones y actos que acontecen en su vida familiar (Rodríguez 
Menéndez, Peña y Torío, 2010). El reparto del trabajo doméstico, según Aasve, Fuochi y Mencarini (2014) se realiza atendiendo a los siguientes aspectos: Ios recursos relativos, la disponibilidad de tiempo, el modelo de dependencia económica y la ideología de género. Ahora bien, estas negociaciones no son equitativas ni neutras, ya que entran en juego diversos factores asociados a las atribuciones de poder de cada miembro de la pareja. De modo que las parejas que presentan una disponibilidad de tiempo e ingresos similares se muestran más igualitarios en la asignación de tareas, por el contrario, cuanto mayor es la desproporción de los ingresos maritales, más desigualdad existe (Aasve, Fuochi y Mencarini, 2014).

En tercer lugar, existen asociaciones significativas del género respecto a las preferencias de tareas, mostrando patrones diferenciados. En este sentido, las tareas domésticas asociadas a la limpieza, cocina y cuidado de la ropa son las más desagradables, las más resistentes al cambio y las que se asocian tradicionalmente a las mujeres (Altintas y Sullivan, 2016). Los hombres no solamente hacen menos tareas, sino que además son más ocasionales y al aire libre, mientras que las mujeres se ocupan del mantenimiento y organización del hogar, del cuidado de los niños, siendo más probable que sean éstas las que acaben solicitando largas licencias o reducción de jornadas ante el nacimiento de la descendencia (Hook, 2010).

Tomar conciencia de esta desigualdad, y de la necesidad moral, social y cívica de atender las necesidades esenciales de las familias, considerada la célula más básica de las sociedades (Fernández Sangrador, 2008), es una cuestión esencial para su adecuado funcionamiento. Sin embargo, a nivel nacional, los últimos datos sobre el empleo del tiempo del Instituto Nacional de Estadística hacen referencia al año 20092010. De ahí, la necesidad de proliferación de estudios que permitan evidenciar las tendencias imperantes en las familias en lo que respecta al empleo del tiempo en el hogar, qué se entiende y cómo se distribuyen la diversidad de tareas de casa.

La aproximación a la investigación sobre la temática evidencia la predominancia de estudios en el ámbito socioeconómico centrado en lo laboral, principalmente desde la perspectiva de los adultos. Sin embargo, la incorporación masiva de las mujeres al mercado laboral y los cambios en la configuración de las familias ha contribuido al cuestionamiento de la división hombres proveedores/mujeres cuidadoras, pero no ha cambiado la distribución tradicional de las tareas domésticas por género (Gómez Urrutia, Arellano Faúndez y Valenzuela Contreras, 2017). En este sentido el informe del Banco de España de $2018^{1}$ aconseja facilitar la conciliación y la corresponsabilidad como medidas para compensar la falta de natalidad, evidenciando el papel de las familias en la sociedad.

Tampoco la diversidad de autores que en las últimas décadas evidencian esta desigualdad, han contribuido a mermar la diferenciación de roles en el ámbito privado familiar, pues como señala Beck \& Beck Gernsheim (2002, p.189) la participación de los hombres en las tareas domésticas sigue siendo muy baja, incluso entre las generaciones más jóvenes. En esta misma línea, Malonda et al. (2017) reconoce que la sociedad española ha evolucionado respecto a los estereotipos de género de forma similar a otros países, y que predomina un fuerte deseo de igualdad como principio rector del desarrollo humano, sin embargo, las adolescentes continúan haciendo más tareas domésticas que los niños. Las nuevas generaciones que se forman en contextos familiares caracterizadas por la desigualdad de género en el reparto de tareas reproducen los roles tradicionales, pues tanto padres como madres acaban 
demandando más colaboración de las hijas en la realización de las tareas domésticas (Wood \& Eagly, 2010; 2012).

Por otra parte, las tareas domésticas son responsabilidad de todos aquellos que conviven en la unidad familiar, no solo de los adultos. En este sentido, la implicación de los infantes-adolescentes a las rutinas familiares, incluidas las tareas domésticas, se considera una actuación formativa saludable para su desarrollo. Pero en contextos empobrecidos, el trabajo doméstico infantil-adolescente se incrementa considerablemente, incluyendo en ocasiones la ayuda en tareas artesanales, agrícolas o ganadera, el cuidado de los hermanos menores y mantenimiento de la vivencia, 0 incluso realizando tareas domésticas en casas ajenas al hogar familiar (Pérez Descalzo y Guijarro Ojeda, 2019), hasta el punto de naturalizar este tipo de prácticas (Anderson, 2007; Cerda, Chávez y Carpio, 2017) que en algunos casos entra en conflicto con los derechos del niño. Esta realidad del trabajo infantil-adolescente doméstico también muestra desigualdad de género, no solo en cuanto la carga, sino también en lo que respecta a los efectos negativos en el desempeño escolar, ya que solo fueron significativos en las adolescentes (Wichmann et al., 2019).

Ante esta realidad se plantean los siguientes interrogantes ¿qué elementos son los que contribuyen a mantener esta división de roles en las tareas domésticas?, ¿qué percepción tienen los hijos de las tareas domésticas?, ¿cómo se implican los diferentes miembros de la familia en la diversidad de tareas que se contemplan en el hogar?, ¿cómo valoran la implicación de sus padres en la educación?

\section{Objetivos}

La presente investigación parte de un doble objetivo. Por un lado, se analiza la visión del alumnado en cuanto a las tareas domésticas llevadas a cabo en el núcleo familiar, para profundizar en los roles asumidos por los miembros de la familia. Por otro lado, se valora la implicación e interés de los padres por la educación de los alumnos, de nuevo en función de la percepción del estudiante, diferenciando entre la opinión según el género del alumno, la edad de los padres y, por último, el nivel de estudios de los padres.

\section{Métodos}

\section{Diseño metodológico}

La investigación realizada es cuantitativa evaluativa, no experimental, con carácter exploratorio.

\section{Participantes}

La muestra del estudio estuvo compuesta por 44 estudiantes de ESO y Bachiller, de edades comprendidas entre los 12 y los 17 años. El criterio de recopilación de la muestra no podía apoyarse en sistemas de selección aleatoria, por ello, la estrategia de muestreo fue no probabilístico intencional.

\section{Procedimiento y técnicas de recogida de información}

La técnica empleada ha sido la encuesta. Concretamente, el instrumento utilizado ha sido un cuestionario para recoger información de la realidad de las diferentes situaciones familiares. 
Al igual que los cuestionarios de usos de tiempo, los referentes a actividades domésticas tienen como objetivo describir la situación respecto a la distribución del tiempo entre los miembros de la unidad familiar, con el fin de aplicar una nueva mirada a la realidad, desde la perspectiva de las ciencias sociales, lo que supone aplicar instrumentos conceptuales y metodológicos capaces de dar cuenta de la dedicación de padres e hijos a las responsabilidades domésticas.

Dicho cuestionario fue construido a partir de diferentes fuentes: Cuestionario sobre conciliación de la vida personal laboral y familiar; Encuesta sobre Solidaridad Familiar; Encuesta sobre pareja y familia en el proyecto vital de los jóvenes; Encuesta a nuevas familias en nuevos municipios; Encuesta pareja y familia en el horizonte vital de las nuevas generaciones.

Para la validación del instrumento se procedió a una revisión por parte de expertos a través del método Delphi. El equipo de expertos estuvo compuesto por cinco profesionales de la educación, procedentes de tres universidades diferentes (Universidad de Murcia, Universidad Católica de Murcia, y Universidad de Oviedo). El cuestionario fue sometido a cuatro rondas de evaluación por los jueces.

Este instrumento concretamente consta de un total de 20 cuestiones. Las cinco primeras son preguntas referidas a variables sociodemográficas, tales como: género del alumno junto con la edad y el nivel de estudios de ambos padres. Las quince siguientes son interrogantes referidos a la percepción del estudiante sobre las tareas domésticas y a la implicación e interés que muestran los padres hacia los asuntos relativos a su proceso educativo.

\section{Técnicas de análisis de datos información}

Para el estudio de la información recabada, proporcionada por el cuestionario aplicado, siguiendo en todo momento un proceso sistemático y riguroso de clasificación y análisis de los datos obtenidos con el paquete estadístico SPSS versión 24.0, se empleó la estadística descriptiva e inferencial paramétrica, una vez reunidas las condiciones necesarias para su aplicación (Siegel, 1991). Así, se estudió la distribución normal de la población con la prueba de Kolmogorov-Smirnov y la homocedasticidad, a través de la prueba de Levene, teniendo en cuenta la condición de «muestra superior a treinta individuos». Como no se encontró significación estadística, se aplicó estadística paramétrica, para comprobar la existencia de diferencias significativas entre los distintos grupos, en relación con las variables seleccionadas. Concretamente, se calculó la prueba $t$ de Student, en el caso de las diferencias en género; Anova de un factor para el estudio de diferencias por edad y nivel de estudios, además de la prueba de comparaciones múltiples de Bonferroni, para evidenciar los grupos entre los que se daban las diferencias significativas. En todos los casos se utilizó un nivel de significación estadística de $\alpha=, 05$. Sin embargo, dado que la significación estadística no proporciona información sobre la fuerza de dicha diferencia, se consideró oportuno estimar la magnitud de la diferencia entre variables, a partir del denominado tamaño del efecto 0 d de Cohen (Cohen, 1988).

\section{Resultados}

A continuación, en respuesta al primer objetivo, relativo a las tareas domésticas en el núcleo familiar, se presentan las puntuaciones, expresadas en porcentajes (\%), de las variables de la investigación. 
Tal y como se aprecia en la Tabla 1, cuando en la familia se habla de tareas del hogar, los estudiantes afirman que, de manera habitual, se hace alusión principalmente al mantenimiento de este $(84,1 \%)$.

Tabla 1. Porcentajes sobre el significado en la familia de tareas del hogar, según el estudiante.

\begin{tabular}{lccc}
\hline ¿Qué se entiende por... & Provisión de recursos & Mantenimiento del hogar & Crianza \\
\hline ...tareas del hogar? & 9,1 & 84,1 & 6,8 \\
\hline
\end{tabular}

En la Tabla 2 se puede observar que los alumnos aseguran que estás tareas son organizadas, en mayor medida, por la figura materna (75\%), la cual a su vez es la persona que mayor número de estas realiza $(88,6 \%)$.

En cuanto al cuidado de los hijos, los estudiantes de nuevo indican que es la madre quien se hace cargo de estos (52,3\%), aunque de manera compartida en otras ocasiones con el padre (29,5\%).

\begin{tabular}{lccccc}
\hline \multicolumn{2}{l}{ Tabla 2. Porcentajes sobre la responsabilidad de las tareas del hogar } & & & \\
\hline ¿Quién se encarga... & Padre & Madre & Ambos & Hijos & Todos \\
\hline ... de organizar las tareas del hogar? & 0 & 75,0 & 13,6 & 4,5 & 6,8 \\
\hline ... de realizar mayor número de ellas? & 2,3 & 88,6 & 0 & 4,5 & 4,5 \\
\hline ...del cuidado de los hijos? & 2,3 & 52,3 & 29,5 & 13,6 & 2,3 \\
\hline
\end{tabular}

Más pormenorizadamente, en la Tabla 3 se muestra quién se encarga de las tareas más relevantes. Así, tal y como se observa, el alumnado cree que la provisión de recursos básicos (alimentación, higiene, ropa, cuidados médicos, etc.) es principalmente llevada a cabo por la figura materna $(68,2 \%)$, aunque también es compartida por ambos cónyuges $(25 \%)$. Pese a ello, el liderazgo y la toma de decisiones se le atribuyen a padre $(43,2 \%)$, o a ambos en todo caso $(40,9 \%)$. Ambas figuras, también, llevan a cabo la comunicación con los miembros de la familia extensa, amigos, compañeros, etc. $(59,1 \%)$. Aunque vuelve a ser la madre la encargada, en la mayoría de los casos, de supervisar y controlar el cumplimento de las responsabilidades asignadas (52,3\%), el mantenimiento y organización del hogar (75\%), el cuidado de los pequeños, mayores o dependientes $(72,7 \%)$ y asegurar que las cosas están preparadas para el día siguiente, decidir sobre limpieza, asignar tareas, etc. (75\%).

\begin{tabular}{lcccc}
\hline \multicolumn{7}{l}{ Tabla 3. Porcentajes sobre el reparto de responsabilidades de cada tarea del hogar } \\
\hline ¿Quién se encarga de... & Padre & Madre & Ambos & Hijos \\
\hline $\begin{array}{l}\text {...la provisión de recursos básicos, tales como alimentación, higiene, } \\
\text { ropa, cuidados médicos? }\end{array}$ & 2,3 & 68,2 & 25,0 & 4,5 \\
\hline ...el liderazgo y la toma de decisiones? & 43,2 & 13,6 & 40,9 & 2,3 \\
\hline $\begin{array}{l}\text {...la comunicación con los miembros de la familia extensa, amigos, } \\
\text { compañeros, etc.? }\end{array}$ & 9,1 & 9,1 & 59,1 & 22,7 \\
\hline $\begin{array}{l}\text {...supervisar y controlar el cumplimento de las responsabilidades } \\
\text { asignadas? }\end{array}$ & 6,8 & 52,3 & 38,6 & 2,3 \\
\hline ...el mantenimiento y organización del hogar? & 4,5 & 75,0 & 15,9 & 4,5 \\
\hline ...el cuidado de los pequeños, mayores o dependientes? & 0 & 72,7 & 25,0 & 2,3 \\
\hline $\begin{array}{l}\text {...asegurar que las cosas están preparadas para el día siguiente, } \\
\text { decidir sobre limpieza, asignar tareas, etc.? }\end{array}$ & 4,5 & 75,0 & 13,6 & 6,8 \\
\hline
\end{tabular}

Haciendo alusión al segundo objetivo, sobre la valoración de la implicación e interés de los padres por la educación de los alumnos, según la percepción de este último, se presentan a continuación las puntuaciones medias $(\tilde{X})$, desviaciones típicas $(\sigma)$, significación estadística $(p)$ y tamaño del efecto $(d)$, de las variables de la investigación. 
Tal y como se aprecia en la Tabla 4, los alumnos consideran que sus familiares directos se interesan bastante por su educación, aunque, concretamente, aseguran que son las madres las que lo hacen en mayor medida $\left(\tilde{X}_{M}=4,39\right)$ que los padres $\left(\tilde{X}_{P}=4,02\right)$.

\begin{tabular}{ccc}
\hline Tabla 4. Estadísticos descriptivos del interés educativo de los padres & & \\
\hline Interés global por la educación & Padre & Madre \\
\hline$\tilde{X}$ & 4,02 & 4,39 \\
\hline$\sigma$ & 1,089 &, 754 \\
\hline
\end{tabular}

En cuanto a la implicación o interés de los familiares en la educación de sus hijos, en función del género del estudiante, se observa en la Tabla 5, que tanto alumnos como alumnas, están de acuerdo en el interés que muestran los padres, y el que muestran las madres, no existiendo diferencias estadísticamente significativas entre dichas puntuaciones otorgadas ( $p_{\mathrm{P}}=, 069 ; p_{\mathrm{M}}=, 145$, respectivamente).

\begin{tabular}{lcccc}
\hline \multicolumn{2}{l}{ Tabla 5. Estadísticos del interés educativo de los padres, según el género del alumno } \\
\hline \multicolumn{7}{l}{ Interés educativo } & Padre & \multicolumn{3}{c}{ Madre } \\
\hline Género & Alumnos & Alumnas & Alumnos & Alumnas \\
\hline$\tilde{X}$ & 3,65 & 4,26 & 4,18 & 4,52 \\
\hline$\sigma$ & 1,222 &, 944 &, 883 &, 643 \\
\hline$p$ & \multicolumn{5}{c}{, 069} & &, 145 & \\
\hline
\end{tabular}

Haciendo alusión a los resultados mostrados en la Tabla 6, los alumnos aseguran que, indiferentemente de la edad que tengo el padre, su nivel de implicación educativo continúa siendo bastante adecuado $\left(\tilde{X}_{P<40}=4,33 ; \tilde{X}_{P 40-50}=3,98 ; \tilde{X}_{P>50}=4,27\right)$, sin existir

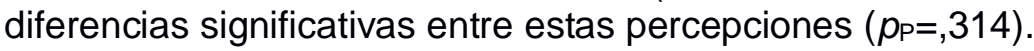

En cambio, la preocupación que creen los hijos que muestran las madres, sí varía significativamente $\left(p_{\mathrm{M}}=, 036\right)$ en función de la edad de las mismas. Concretamente, son las madres más jóvenes las que presentan menor índice de interés $\left(\tilde{X}_{M<40}=3,81\right)$ en las cuestiones escolares, que las madres más mayores $\left(\tilde{X}_{M 40-50}=4,61 ; \tilde{X}_{M>50}=4,40\right)$, encontrándose diferencias estadísticamente significativas entre las primeras y las que

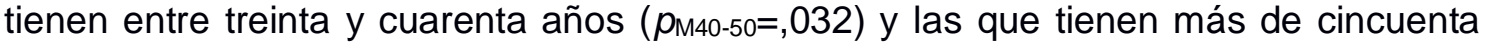

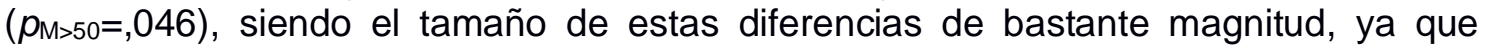
arrojan ambos valores muy por encima del índice establecido por Cohen $\left(d_{\mathrm{M} 40-50}=1,121\right.$; $d_{\mathrm{M}>50=, 841) \text {. }}$

\begin{tabular}{|c|c|c|c|c|c|c|}
\hline \multirow{2}{*}{$\begin{array}{l}\text { Interés educativo } \\
\text { Edad }\end{array}$} & \multicolumn{3}{|c|}{ Padre } & \multicolumn{3}{|c|}{ Madre } \\
\hline & $<40$ & $40-50$ & $>50$ & $<40$ & $40-50$ & $>50$ \\
\hline$\tilde{X}$ & 4,33 & 3,98 & 4,27 & 3,81 & 4,61 & 4,40 \\
\hline$\sigma$ & 816 & 1,313 & ,704 & ,701 & ,722 & ,699 \\
\hline$p$ & \multicolumn{3}{|c|}{,314 } & \multicolumn{3}{|c|}{, 036} \\
\hline
\end{tabular}

Por último, tras evaluar el interés de los padres en función del nivel de estudios que poseen, se muestra en la Tabla 7, que al igual que en el supuesto anterior, los alumnos afirman que el interés del padre no depende en demasía del nivel formativo que el mismo posea $\left(\tilde{X}_{P . E S O}=3,91 ; \tilde{X}_{\text {P.BACH}}=4,42 ; \tilde{X}_{\text {P.UNIV }}=4,20\right)$, sin existir, de nuevo, diferencias significativas entre dichas opiniones $\left(p_{\mathrm{P}}=, 266\right)$.

Sin embargo, a reflejo nuevamente de lo ocurrido con anterioridad, la implicación que creen los hijos que muestran las madres, sí varía significativamente $\left(p_{\mathrm{M}}=, 036\right)$ en función de la edad de las mismas. Concretamente, son las madres sin estudios las menos interesadas $\left(\tilde{X}_{M . S I N}=3,10\right)$ en lo relativo a las cuestiones escolares, frente a las madres con formación académica $\left(\tilde{X}_{M . E S O}=4,61 ; \tilde{X}_{M . B A C H}=4,50 ; \tilde{X}_{M . U N I V}=4,26\right)$. En esta casuística 
también se hallan diferencias estadísticamente significativas $\left(p_{\mathrm{M}}=, 000\right)$, concretamente

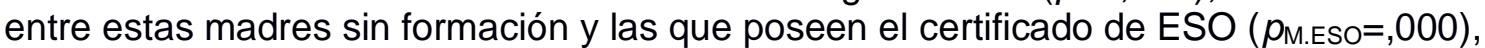
las que tienen el título de Bachiller $\left(p_{\mathrm{M} . \mathrm{BACH}}=, 001\right)$ y las que poseen titulación universitaria $\left(p_{\text {M.UNIV }}=, 048\right)$. Siendo de nuevo el tamaño del efecto de estas diferencias bastante considerable, ya que arrojan todas las puntuaciones, valores muy por encima del índice

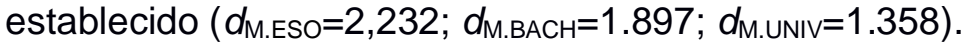

\begin{tabular}{|c|c|c|c|c|c|c|c|c|}
\hline \multicolumn{2}{|c|}{ Interés educativo } & \multicolumn{3}{|c|}{ Padre } & \multicolumn{4}{|c|}{ Madre } \\
\hline Estudios & Sin estudios & ESO & Bach. & Univ. & Sin estudios & ESO & Bach. & Univ. \\
\hline$\tilde{X}$ & - & 3,91 & 4,42 & 4,20 & 3,10 & 4,61 & 4,50 & 4,26 \\
\hline$\sigma$ & - & 1,241 & 669 & 837 & 816 &, 499 & 650 & 890 \\
\hline$p$ & \multicolumn{4}{|c|}{266} & \multicolumn{4}{|c|}{, 000} \\
\hline
\end{tabular}

\section{Conclusiones, consecuencias e implicaciones}

Tal y como se ha visto reflejado en el apartado de resultados de la investigación, en respuesta al primer objetivo, relativo a las tareas domésticas en el núcleo familiar, la inmensa mayoría de los estudiantes afirma que cuándo en su familia se habla de tareas del hogar, se hace alusión a labores de limpieza y no a situaciones como la provisión de recursos o la crianza en sí. Esto indica que no incluyen como tarea doméstica lo concerniente a la educación y cuidado, aunque ambas son parte integrante de las responsabilidades familiares.

En general, el trabajo doméstico no es deseable y las personas intentan evitarlo (Sullivan, 2011), incrementando los reproches y desacuerdos en la pareja. Por un lado, ellas se quejan de la escasa participación de sus parejas, por otro, ellos recriminan que sus mujeres son muy exigentes en el desempeño doméstico y les cuesta delegar (Rodríguez Menéndez, Peña Calvo y Torío López, 2010). Si bien la experiencia del trabajo doméstico ya no es ajena a los hombres, los resultados obtenidos muestran que la figura de la madre se erige como centro de planificación y distribución de tareas, llevando estas a cabo en un mayor número de ocasiones que el padre, según el alumnado. La fuerza de los convencionalismos de género todavía se encuentra presente en las familias, de modo que para "las mujeres las responsabilidades domésticas son prioritarias, mientras que los varones, aunque participen, son conscientes, y lo asumen, de que desempeñan un papel secundario" (Rodríguez Menéndez, Peña Calvo y Torío López, 2010, p.114), y esta desigualdad "constituye uno de los obstáculos fundamentales en el avance hacia una sociedad sin discriminación de género" (Royo, 2011, p.16).

Los estudiantes de nuevo indican que es la madre quien se hace cargo del cuidado de los más pequeños, mayores o dependientes, además de mantenimiento, organización y provisión de recursos básicos en el hogar, supervisión y control del cumplimento de las responsabilidades asignadas a cada uno y toma de decisiones sobre limpieza o asignación de tareas. Pese a ello, el liderazgo y la toma de decisiones se le atribuyen principalmente al padre, lo que brinda una perspectiva de pensamiento clásico de las generaciones jóvenes a favor de posiciones en desequilibrio con respecto a las madres, concediendo la autoridad al rol paterno, pese a que son ellas paradójicamente quienes, a su juicio, atienden el mantenimiento de la casa y crianza.

Haciendo alusión al segundo objetivo, referido a la valoración de la implicación e interés de los padres y madres por la educación de los alumnos, según la percepción de estos últimos, se ha podido comprobar que defienden que sus progenitores se interesan bastante por su educación, aunque aseguran que son las madres las que lo hacen en 
mayor medida. En consonancia, de forma general se observan diferencias de género en lo que respecta al cuidado de los menores, especialmente en el cuidado físico y atención de las necesidades básicas (Flaquer et al, 2019). Además, el análisis de la implicación de los progenitores, en función del género del estudiante, denota que tanto alumnos como alumnas se posicionan de acuerdo en el interés que muestran los padres, y el que muestran las madres, no existiendo diferencias estadísticamente significativas entre dichas puntuaciones. Ambos reconocen la existencia de esta diferenciación de género respecto al cuidado de los hijos.

Por otra parte, indiferentemente de la edad que tenga el padre, su nivel de implicación educativo continúa siendo bastante adecuado, de nuevo sin arrojar diferencias significativas estas percepciones. En cambio, la preocupación que creen los hijos que muestran las madres, sí varía significativamente en función de la edad de las mismas. Concretamente, son las madres más jóvenes las que presentan menor índice de interés por las cuestiones escolares, hallándose diferencias estadísticamente significativas entre las primeras y las que tienen entre treinta y cuarenta años y las que tienen más de cincuenta, siendo el tamaño de estas diferencias de bastante magnitud, ya que arrojan ambos valores muy por encima del índice establecido por Cohen. En esta misma línea, los perfiles de participación familiar en Educación Infantil, Primaria y Secundaria descritos por Parra, Hernández Prados, Gomáriz y García Sanz (2015), evidencian que las familias de padres y madres más jóvenes, de nacionalidad española, con menor nivel de estudios y cuyos hijos asisten a centros públicos presentan una menor implicación en las cuestiones escolares con índices bajos en sentimiento de pertenencia, asistencia a las actividades de centro e implicación desde el hogar.

Por último, de nuevo los alumnos afirman que el interés por los asuntos escolares del padre no depende en demasía del nivel formativo que el mismo posea, sin existir diferencias significativas entre dichas opiniones. Sin embargo, nuevamente la implicación que creen los hijos que muestran las madres, sí varía significativamente en función de la formación de las mismas. Concretamente, son las madres sin estudios las menos interesadas en las cuestiones escolares, frente a las madres con formación académica. En esta casuística también se hallan diferencias estadísticamente significativas, concretamente entre estas madres sin formación y las que poseen el certificado de ESO, las que tienen el título de Bachiller y las que poseen titulación universitaria. Siendo de nuevo el tamaño del efecto de estas diferencias bastante considerable, ya que arrojan todas las puntuaciones, valores muy por encima del índice establecido. En relación a lo expuesto, el estudio de revisión empleando datos transnacionales realizado por Sullivan, Billari y Altintas (2014) evidencia de manera significativa una mayor contribución a las tareas domésticas y al cuidado de los hijos en los progenitores más jóvenes y con mayor nivel de estudios.

Concluimos este estudio trayendo a coalición las palabras de Díaz Gorfinkiel (2019) que dicen así: "la construcción de los nuevos roles masculinos y femeninos se haya en proceso y genera, en consecuencia, dificultades conceptuales y de puesta en práctica de las nuevas nociones de género e igualdad" (p.220). Por tanto, aún queda un largo camino por recorrer en el esclarecimiento de las desigualdades de género y la influencia educativa de las dinámicas intrafamiliares en la socialización de las responsabilidades familiares asociadas a las tareas domésticas y el cuidado de la descendencia. 


\section{Referencias}

Aasve, A., Fuochi, G \& Mencarini, L (2014). Desperate Housework: Relative Resources, Time Availability, Economic Dependency, and Gender Ideology across Europe. Journal of Family Issues, 35(8), 1000-1022.

Alberdi, I. (2014). El retraso de la procreación y el mantenimiento de la juventud. Metamorfosis, 10, 9-25

Diaz Gorfinkiel, M. (2019). La conciliación en la encrucijada: vida laboral y familiar de empleadas y empleadoras de hogar. Lex Social: Revista de Derechos Sociales, 9(2), 198-211.

Altintas, E., \& Sullivan, O. (2016). Fifty years of change updated: Cross-national gender convergence in housework. Demographic Research, 35, 455-470.

Anderson, J. (2007). Invertir en la Familia: Estudio sobre factores preventivos y de vulnerabilidad al trabajo infantil doméstico en familias rurales y urbanas de Colombia, Paraguay y Perú. Lima: OIT.

Aspiazu, E. (2014). Conciliación entre trabajo y responsabilidades familiares: una revisión teórica con enfoque de género. Investigium IRE: Ciencias Sociales y Humanas, 1, 177-194.

Beck, U., \& Beck Gernsheim, E. (2002). Individualization: Institutionalized individualism and its social and political consequences. London: Sage.

Cerda, A., Chávez, M. y del Carpio, P. (2017). Reflexiones sobre el trabajo infantil. Jóvenes en la ciencia. Revista De Divulgación Científica, 3(2), 62-66.

Cerri, C. y Alamillo Martínez, L. (2012). La organización de los cuidados, más allá de la dicotomía entre esfera pública y esfera privada. Gazeta de Antropología, 28(2), artículo 14.

Cohen, J. (1988). Statistical Power Analysis for the Behavioral Sciences ( $2^{\mathrm{a}}$ Edición). Academic Press.

De Dios Herrero, M. (2019). Las tareas de cuidado en Roma.: Miradas al interior de la familia. La Aljaba: Segunda Época, Revista de Estudios de la Mujer, 23, 81-90.

Devetter, F.X. (2013). ¿Por qué externalizar las tareas domésticas? Análisis de las lógicas desigualitarias que estructuran la demanda en Francia. Revista de Estudios Sociales, (45), 8095.

Fernández Sangrador, J. (2008). La familia, célula vital de la sociedad. La Revue du redif, 1, 5-7.

Flaquer, L., Navarro Varas, L., Antón-Alonso, F., Ruiz Forès, N. y Cónsola, A. (2019). La implicación paterna en el cuidado de los hijos en España antes y durante la recesión económica. Revista Española de Sociología, 28(2)

Garavito, C. (2018). Demanda de trabajo del hogar remunerado en el Perú urbano. Economía, 41(82), 35-60.

García Román, J. (2020). La división de los roles de género en las parejas en las que solo trabaja la mujer en Estados Unidos y España. REIS: Revista Española de Investigaciones Sociológicas, (170), 73-94.
Gómez Urrutia, V., Arellano Faúndez, O. y Valenzuela Contreras, C. (2017). Negociaciones en familia: género, trabajo y cuidado en Chile. Revista Estudios Feministas, 25(2), 661-682.

Hernández Prados, MA. y Lara Guillen, B. (2015) Responsabilidad familiar ¿una cuestión de género? RES, Revista de Educación Social, 21, 28-44.

Hook, JL. (2010). Gender inequality in the welfare state: Sex segregation in housework, 1965-2003. American Journal of Sociology, 115, 1480-1523.

Instituto de la Mujer (2013). Mujeres en cifras. Boletín estadístico. http://www.inmujer.gob.es/estadisticas/boletinEstadistico/docs Boletin3Marzo2013.pdf

INE. (2010). Encuesta de empleo del tiempo. Madrid: Instituto Nacional de Estadística. https://www.ine.es/dyngs//NEbase/es/operacion.htm?c=Estad istica_C\&cid=1254736176815\&menu=resultados\&idp $=12547$ 35976608\#!tabs-1254736194826

Malonda, E., Tur Porcar, A. y Llorca, A. (2017). Sexismo en la adolescencia: estilos de crianza, división de tareas domésticas, conducta prosocial y agresividad. Revista de Psicología Social, 32(2), 333-361.

Parra, J., Hernández, M.A., Gomáriz, MA. y García Sanz, MP. (2015). Características de la participación de las familias en la educación escolar. En Consejo Escolar de la Región de Murcia. XIII Encuentro del Consejo Escolar de la Región de Murcia. La participación de las familias en la educación (1133). Murcia: CERM.

Pérez Descalzo, M., \& Guijarro Ojeda, J.R. (2019). Desafío en la enseñanza de español/L2 en comunidades quechua. ReiDoCrea - Monográfico sobre Perspectivas transnacionales en la enseñanza de lenguas, 8(3),191-201. https://www.ugr.es/ reidocrea/8.3-11.pdf

Región de Murcia (2019). Estrategia 2019-2021 para la eliminación de la brecha salarial en la Región de Murcia. http://hdl.handle.net/20.500.11914/3702

Rodríguez Menéndez, MC., Peña Calvo, JV. y Torío López, S. (2010). Corresponsabilidad familiar: negociación e intercambio en la división del trabajo doméstico. Papers: Revista de sociología, 95(1), 95-117.

Royo, R. (2011). Maternidad, paternidad y conciliación en la CAE. ¿Es el trabajo familiar un trabajo de mujeres? Bilbao: Universidad de Deusto. http://www.izenpe.eus/s154812/es/contenidos/informacion/pub publicaciones/es def/ad juntos/6904-maternidad.pdf

Sánchez Vargas, A., Herrera Merino, AL. y Perrotini Hernández, I. (2015). La participación laboral femenina y el uso del tiempo en el cuidado del hogar en México. Contaduría y administración, 60(3), 651-662.

Sieguel, S. (1991). Estadísticos no paramétricos aplicada a las ciencias de la conducta ( $3^{\text {a }}$ Edición). Editorial Trillas. 
Sullivan, O. (2011). An end to gender display through the performance of housework? A review and reassessment of the quantitative literature using insights from the qualitative literature. Journal of Family Theory \& Review, 3, 1-13.

Sullivan, O., Billari F., \& Altintas, E. (2014). Fathers' changing contributions to childcare and domestic work in very low-fertility countries: The effect of education. Journal of Family Issues, 35(8), 1048-1065.

Wichmann, F., Dellazzana Zanon, LL., De Lucca Freitas, LB. y Teixeira, MAP. (2019). Relações entre projetos de vida, tarefas domésticas e desempenho escolar em adolescentes. Revista Psicologia-Teoria e Prática, 21(1).

Wood, W., \& Eagly, AH. (2010). Gender. In ST. Fiske, DT. Gilbert, \& G. Lindzey (Eds.), Handbook of social psychology (pp. 629667). Hoboken: Wiley.

Wood, W., \& Eagly, AH. (2012). Biosocial construction of sex differences and similarities in behavior. Advances in Experimental Social Psychology, 46, 55-123. 